\title{
Modification of Low Refractive Index Polycarbonate for High Refractive Index Applications
}

\author{
Gunjan Suri, Gouri Shankar Jha, Geetha Seshadri, and Rakesh Kumar Khandal
}

Shriram Institute for Industrial Research, 19 University road, Delhi 110007, India

Correspondence should be addressed to Rakesh Kumar Khandal, rkhandal@shriraminstitute.org

Received 19 March 2009; Revised 21 July 2009; Accepted 2 September 2009

Recommended by Jani Matisons

\begin{abstract}
Polycarbonates and polythiourethanes are the most popular materials in use today, for optical applications. Polycarbonates are of two types which fall in the category of low refractive index and medium refractive index. The present paper describes the conversion of low refractive index polycarbonates into high refractive index material by the use of a high refractive index monomer, polythiol, as an additive. Novel polycarbonates, where the properties of refractive index and Abbe number can be tailor made, have been obtained. Thermal studies and refractive index determination indicate the formation of a new polymer with improved properties and suitable for optical applications.
\end{abstract}

Copyright (C) 2009 Gunjan Suri et al. This is an open access article distributed under the Creative Commons Attribution License, which permits unrestricted use, distribution, and reproduction in any medium, provided the original work is properly cited.

\section{Introduction}

Amongst the optical polymers used so far for optical applications, the most popular ones belong to polycarbonate chemistry. Polycarbonates currently used for optical applications are classified into two types based on the two different monomers produced by reacting carbonyl chloride (phosgene) with two different diols [1-5]. Depending upon the characteristics of the diol used, the polycarbonates of different properties can be obtained. For the last five decades, when for the first time, polycarbonates were developed for making spectacle lenses, there have been attempts to not only develop better materials than polycarbonates but also to develop a process which can avoid the use of phosgene. A number of polycarbonates have been developed ever since. However, only two types of polycarbonates are being used for spectacle lenses: (i) Diethylene glycol di(allyl carbonate) (DEGDAC) (free radical polymerization) and (ii) Bisphenol A carbonate (BPAC) (condensation polymerization).

A comparison of different properties of the two types of polycarbonates is presented in Table 1.

The polymerization of DEGDAC involves a radical polymerization process, which can be affected by thermal process [4] or by radiation processing [6], which is found easy by the manufacturers whereas the manufacturing of BPAPC (Bisphenol A polycarbonate) takes place through condensation process [7], which is not feasible for the smallscale manufacturers. That is why, perhaps, DEGDAC is the most popular material for spectacle lenses. Therefore, while the producers of the monomer DEGDAC would find takers for their product (a monomer) across the world, this is not so obvious in the case of BPAPC which is produced directly as a polymer and not as a monomer.

A review [8] of developments on materials for optical plastics suggest that for achieving the objectives of producing the materials of high refractive index as well as high Abbe number, the following approaches have been found prospective, for the future:

(a) completely new chemistry by designing monomers with functional groups and bonds of high refractive index, for example, polythiourethane $[9,10]$ for refractive index $>1.65$, and so forth;

(b) metal-containing polymers utilizing the complementary properties of metals and organic polymers to design materials of desired qualities, for example, optical plastics, based on barium, lanthanum, and lead salts [11-13];

(c) combination of various monomers to obtain synergistic formulations exhibiting the expected characteristics. No reference using this approach has been found so far. 
TABle 1: Properties of two types of polycarbonates, that is, Bisphenol A polycarbonate (BPAPC) and Poly(diethylene glycol di(allyl carbonate)) (PDEGDAC) [2].

\begin{tabular}{lccccc}
\hline Carbonate polymer & Refractive index & Abbe number & $\operatorname{Tg}\left({ }^{\circ} \mathrm{C}\right)$ & Specific gravity & Rockwell hardness $(\mathrm{M})$ \\
\hline PDEGDAC & 1.50 & 59.3 & 85 & 1.30 & 97 \\
BPAPC & 1.59 & 34 & 150 & 1.20 & $70-121$ \\
\hline
\end{tabular}

TABLE 2: Properties of monomers: Diethylene glycol di(allyl carbonate) and polythiol.

\begin{tabular}{lccccc}
\hline Monomer & Refractive index & Appearance & $\%$ SH & Solubility & Specific gravity \\
\hline Diethylene glycol di(allyl carbonate) & 1.45 & Colorless & 0.0 & Acetonitrile & 1.15 \\
Polythiol & 1.69 & Colorless & 36.0 & Toluene & 1.26 \\
\hline
\end{tabular}

In the present case, the aim has been to bring improvements by incorporation of high refractive index monomer, for example, polythiol, in the properties of the most popular polymer of DEGDAC, (PDEGDAC) which is a low refractive index material.

The curiosity as to whether the addition of polythiol to DEGDAC results in a homogeneous material and if yes, then the positive effects that can be achieved by blending of the polythiols with the most popular material, that is, DEGDAC, have been the reason behind this study. The success of this attempt was expected to provide the possibility of an increase in the refractive index of DEGDAC. Further, with varying content of the additive of high refractive index, it would become feasible to tailor make the desired optical properties of DEGDAC. The high refractive index monomer, polythiol, as synthesized by the authors is used as an additive for this study. Considering the fact that for the last fifty years there has been no improvement in DEGDAC, the development of such novel compositions based on this monomer, carried out for the first time, is expected to provide new options for the optical plastics industry.

\section{Materials and Methods}

2.1. Materials: Both the monomers, that is, polythiol (PSH) and diethylene glycol di(allyl carbonate) (DEGDAC) used here are of industrial grade. While DEGDAC, an imported monomer, was procured from Indian Optics, Gurgaon, one of the manufacturers of spectacle lenses in India, polythiol was synthesized by the authors as per the process developed [14-17]. The properties of both the monomers are listed in Table 2.

For polymerization, the catalyst dibutyl tin dilaurate (DBTDL) and Toluene di-isocyanate (TDI) were procured from local sources. The compositions were cast molded into lenses, which were cured by thermal process.

2.2. Methods. PSH and DEGDAC were used for the preparation of modified polycarbonate lenses. The following steps were involved in the preparation of lenses. (a) Degassing: Polythiol was taken alongwith the catalyst DBTDL $(0.5 \%)$ and degassed at $100^{\circ} \mathrm{C}$ for one hour. It was cooled down to subambient temperatures after degassing. DEGDAC was degassed separately at room temperature for 30 minutes.

(b) Cast polymerisation: The degassed DEGDAC and TDI were added gradually to polythiol with continuous stirring at subambient temperatures. For every one mole of PSH used, 2 moles of TDI were added to react with four $-\mathrm{SH}$ groups per mole of PSH. Various ratios of polythiol and DEGDAC as given in Table 3 were prepared. The mixture was injected into glass molds of $60 \mathrm{~mm}$ diameter placed in the grooves $(2 \mathrm{~mm})$ of a gasket. The filled molds were placed in a circulating hot air oven at $60^{\circ} \mathrm{C}$ for a period of 12 hours followed by curing at $90^{\circ} \mathrm{C}$ for a period of four hours. The polymerisation and curing cycle used for this study was as given in Figure 1.

(c) Demolding and evaluation: The cast lenses were demolded from the gaskets to obtain lenses, which were then evaluated for the following properties:

(1) Refractive index and Abbe number: Refractive index and Abbe number of the polymers was carried out on an Atago Refractometer Model: DRM-4 as per ASTM D-542, 1990 [18]. The refractometer is provided with optical filters of wavelength $546 \mathrm{~nm}, 480 \mathrm{~nm}$, and $644 \mathrm{~nm}$ to measure the Abbe number. The samples were rubbed, polished, and made smooth on a polishing wheel at SRI, Delhi. Monobromonaphthalene was used as a contact liquid. Abbe number was calculated using the following equation:

$$
v_{e}=\left(n_{546}-1\right) /\left(n_{480}-n_{644}\right),
$$

where $n_{546}$ is refractive index at $\lambda_{\max }$ of $546 \mathrm{~nm}$ is refractive index at, $\lambda_{\max }$ of $480 \mathrm{~nm}$ and $n_{644}$ is refractive index at $\lambda_{\max }$ of $644 \mathrm{~nm}$.

(2) Specific gravity: Specific gravity of the polymers was determined as per ASTM D-792-00 [19]. 
TABLE 3: Effect of polythiol on the optical properties of diethylene glycol di(allyl carbonate) (DEGDAC).

\begin{tabular}{|c|c|c|c|c|c|c|}
\hline Ratios & Percentage (PSH:DEGDAC) & Refractive index & Specific gravity & Abbe number & Transmittance (\%) & Impact resistant test \\
\hline 1. & $44: 56$ & 1.59 & 1.302 & 32 & 90 & Pass \\
\hline 2. & $47: 53$ & 1.63 & 1.308 & 29 & 90 & Pass \\
\hline 3. & $50: 50$ & 1.61 & 1.263 & 28 & 90 & Pass \\
\hline 4. & $54: 46$ & 1.62 & 1.320 & 27 & 90 & Pass \\
\hline 5. & $58: 42$ & 1.65 & 1.317 & 28 & 90 & Pass \\
\hline 6. & $64: 36$ & 1.64 & 1.345 & 27 & 90 & Pass \\
\hline 7. & $70: 30$ & 1.64 & 1.347 & 28 & 90 & Pass \\
\hline 8. & $78: 22$ & 1.65 & 1.346 & 27 & 90 & Pass \\
\hline 9. & $88: 12$ & 1.66 & 1.354 & 27 & 90 & Pass \\
\hline 10. & $100: 0$ & 1.67 & 1.360 & 28 & 90 & Pass \\
\hline
\end{tabular}

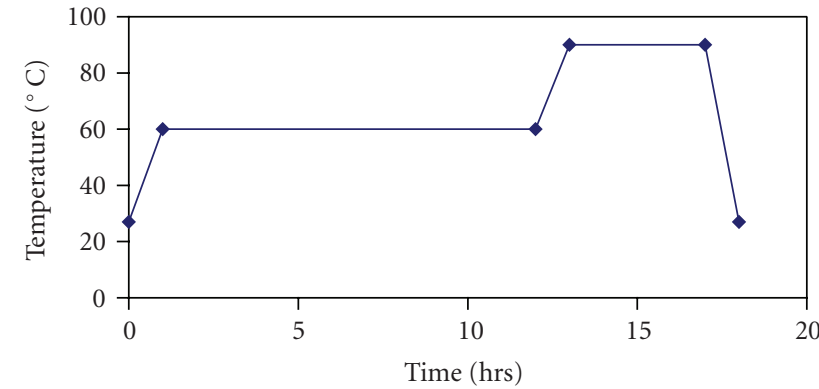

Figure 1: Curing cycle of compositions of PSH and DEGDAC. Transparent and bubble free lenses obtained at the end of the cycle i complete polymerization.

(3) Thermal stability: DSC and TGA/DTA/DTG analysis were carried out on SDT-2960 from TA Waters in the presence of nitrogen at a heating rate of $10^{\circ} \mathrm{C} / \mathrm{min}$.

(4) Transmittance (\%): The transmittance of the cast lenses in the UV-Visible region of $200 \mathrm{~nm}$ to $800 \mathrm{~nm}$ was determined on the UV-Visible spectrophotometer (Model number UV-1700) from Shimadzu.

(5) Impact resistance: 50 lenses of $2 \mathrm{~mm}$ diameter each were tested for impact resistance as per FDA 21 CFR 801.410 [20], where a steel ball weighing $16.2 \mathrm{~g}$ was dropped from a height of $127 \mathrm{~cm}$ upon the horizontal upper surface of the lens. Lenses, which do not break or crack, were taken to be impact resistant and reported as pass.

\section{Results and Discussion}

The advent of plastic materials as a replacement to the conventional material, glass, has so far led to the development of two popular materials, namely, polycarbonates and polyacrylates. Out of the two, it is only polycarbonates, which are suitable for precision optics while polyacrylates are used for nonprescription optics such as sunglasses, and so forth. There has been no material so far which has been found to be superior to polycarbonates. DEGDAC based materials are still the most popular materials in many countries; an attempt to increase the refractive index of this material while retaining other favorable properties of DEGDAC would be an added advantage. The polythiol that has been used in this work has been patented $[14,15]$ by the authors and its use for tailor-making of optical properties has not been reported so far. Moreover, tailor-making of optical properties such as refractive index and Abbe number is not a common phenomenon in the industry. Generally, the ophthalmic lenses are based on a single material without any possibility of tailor-making. For any improvement in refractive index, and so forth. one needs to opt for a completely new chemistry, whereas in the present study, tailor-making of optical properties has been demonstrated for the first time.

The results of the effect of increase in content of polythiol in DEGDAC on various optical properties are presented in Table 3. The values of refractive index, Abbe number, and specific gravity have also been subjected to third-party evaluation.

First of all, it must be noted that the various combinations of the two monomers are homogeneous upto a maximum concentration of $56 \%$ (by weight) of DEGDAC; beyond 56\%, DEGDAC separates out from the polythiol. This means that all the combinations containing more than $44 \%$ (by weight) of polythiol and not less than $56 \%$ by wt. of DEGDAC have been found to be homogeneous. In other words, if DEGDAC is added to polythiol, it will only dissolve upto $56 \%$ of DEGDAC. Thus, the solution of $56 \%$ (by wt.) concentration of DEGDAC in polythiol can be taken as the saturated solution. Further, the addition of diisocyanate and the catalyst (necessary for the formation of the polythiourethane) could also be carried out at all the concentrations below the saturation point of polythiol. This shows that the two monomers are compatible and hence, it is possible to modify the optical properties of DEGDAC by the incorporation of polythiol. The results indicate that the essential optical properties of DEGDAC can be tailor-made to a desired level by using different ratios of PSH:DEGDAC.

The effect of increasing the concentration of polythiol has brought an improvement as seen by an increase in the refractive index and a decrease in the specific gravity upto an optimum ratio of PSH:DEGDAC::50 : 50. However, the addition of polythiol has brought a reduction in the 


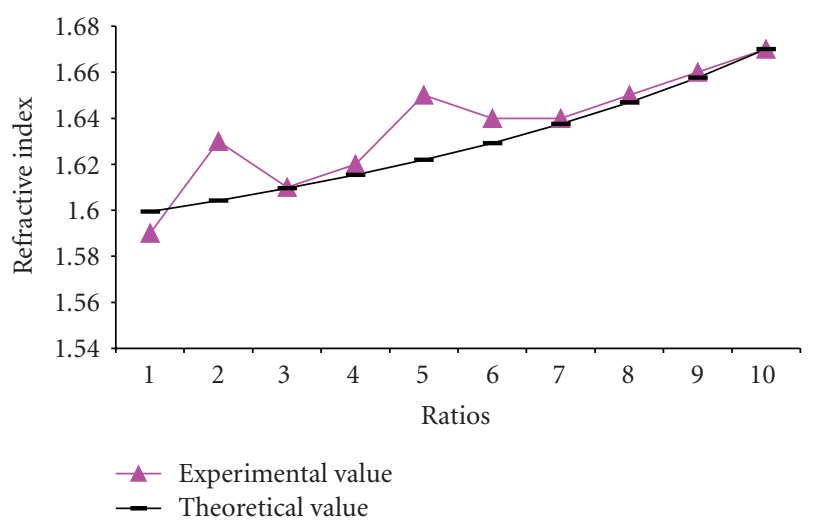

FIGURE 2: Effect of addition of PSH to DEGDAC on the refractive index of PDEGDAC. By varying the concentration of $\mathrm{PSH}$, the refractive index of PDEGDAC can be increased upto 1.66.

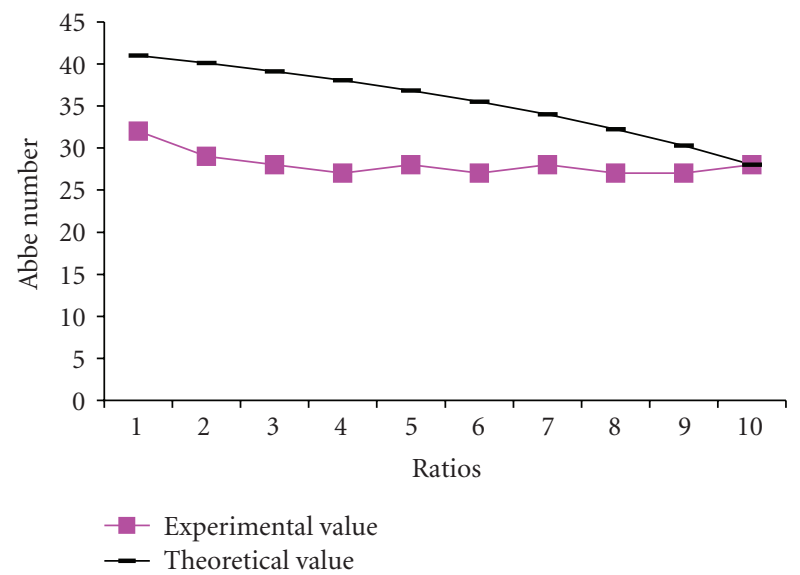

FIgURE 3: Effect of addition of PSH on DEGDAC. Abbe number is maximum for PSH:DEGDAC::44:56 (32) and can be tailor-made in the range of 27 to 32 by changing the concentration of $\mathrm{PSH}$.

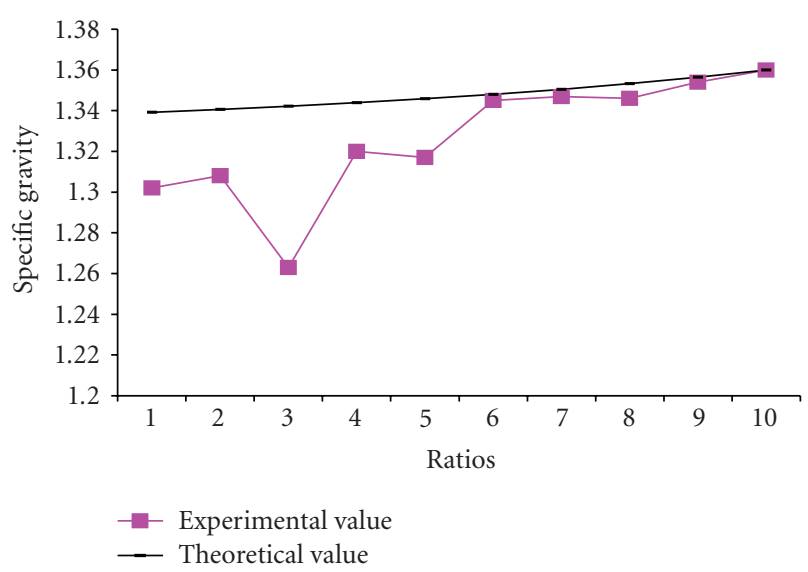

FIGURE 4: Effect of PSH on specific gravity of DEGDAC: Increase in percentage of the PSH increases the specific gravity of the polymer; polymer obtained with a percentage ratio of $50: 50$ of PSH:DEGDAC is found to be the lightest.

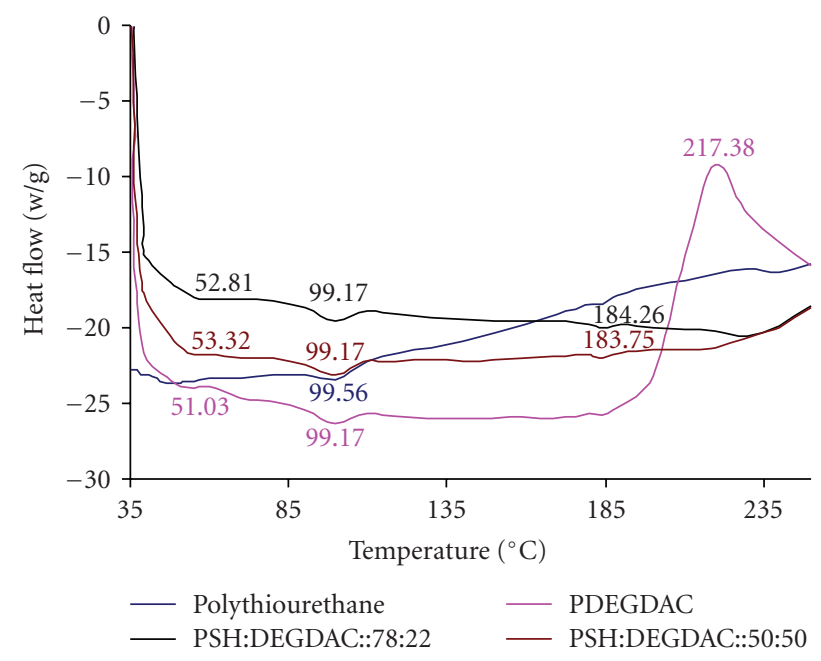

FIGURE 5: DSC thermogram of Polythiourethane, PDEGDAC, and combination of PSH and DEGDAC.

Abbe number from 59 to 32 for the combinations having maximum possible content (56\%) of DEGDAC. Attaining an Abbe number higher than the minimum desired limit of say 30 is possible with the combination of the two monomers studied here. The transmittance and Abbe number of all ratios are found to be meeting the minimum required limits of $90 \%$ for transmittance, and 30 for Abbe number. All the combinations have passed the test for impact resistance. Based on these results, it can be said that the right ratio can be chosen for the purpose of obtaining optical plastics of varying properties.

3.1. Refractive Index. The results of refractive index with increasing concentration of polythiol are shown in Figure 2. The trend in refractive index (theoretical versus experimental) is depicted in Figure 2.

It may be seen that over the range of concentrations studied here, the refractive index increases from 1.49 (for PDEGDAC) to 1.59 (for $44 \%$ of PSH and $56 \%$ of DEGDAC). Further addition of polythiol can result in a polymer with a refractive index of more than 1.59 and even beyond 1.60. This shows that the refractive index of polycarbonate (1.49) falling in the low refractive index range can be increased to more than 1.60 , which is the minimum limit for high refractive index materials. The obtained values are higher and found to follow a different trend than the theoretical values indicating the formation of a different structure. Thus, polycarbonates can be converted into high refractive index materials by just blending DEGDAC with high refractive index materials of different chemistry (thiol based).

3.2. Abbe Number. The results (Figure 3) of Abbe number with increasing concentration of polythiol ranges from 32 (for $44 \%$ polythiol) to 28 (for $100 \%$ polythiol). Abbe number of polycarbonate thus, gets reduced from 59.3 to less than 28 with the addition of polythiol. The ratios can be chosen based on the desired Abbe number. 


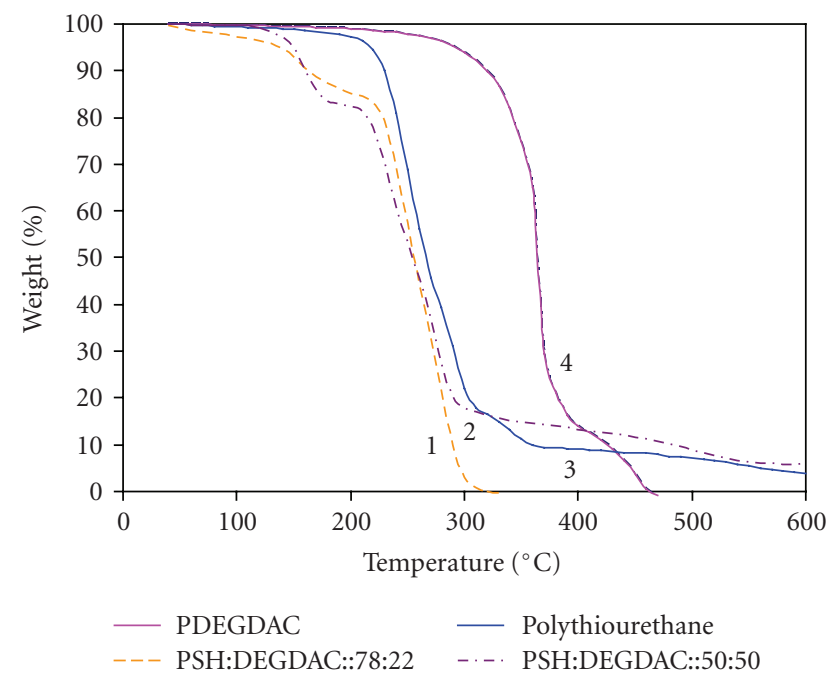

FIgure 6: TGA thermogram of Polythiourethane, PDEGDAC, and combination of PSH and DEGDAC. The results indicate better thermal stability in the product as compared to PDEGDAC.

3.3. Specific Gravity. The results of specific gravity as presented in Figure 4 suggest that there is a distinct drop in specific gravity at ratios of $50: 50$ of the two monomers. The trend in the theoretical values as calculated using the law of mixtures is also depicted. This is unique, as the specific gravity of polymer with 50 : 50 monomer ratio is lower than the specific gravity of both the ingredients. This can only be explained with the reasoning that the combination at the ratio of $50: 50$ forms a structure, which is more compact than the polymers based on the individual components, that is, polycarbonate and polythiourethane. This can again be taken as the criteria for deciding the right ratio for mixtures. The obtained values are much higher than the values obtained from the theoretical law of mixtures indicating the formation of a new structure.

It is evident that there is a positive deviation, that is, higher values than the theoretical values in the case of refractive index and negative deviation, that is, lower values than the theoretical values in the case of Abbe number and specific gravity. This suggests that the combination of the two different polymers is not just a simple mixture wherein the two polymers coexist without any structural change but is a case where changes leading to the formation of a complex structure for which the law of mixture is not applicable takes place.

The values presented here have also been confirmed by the third-party evaluation, by a major manufacturer of lenses.

3.4. Thermal Analysis. In order to understand the polymerisation of the mixtures of DEGDAC and polythiol, DSC, TGA, DTA, and DTG studies were carried out. The results of polymers as such and their combinations (PSH:DEGDAC::50 $: 50,78: 22$ ) are presented in Figures 5, 6, 7, and 8.

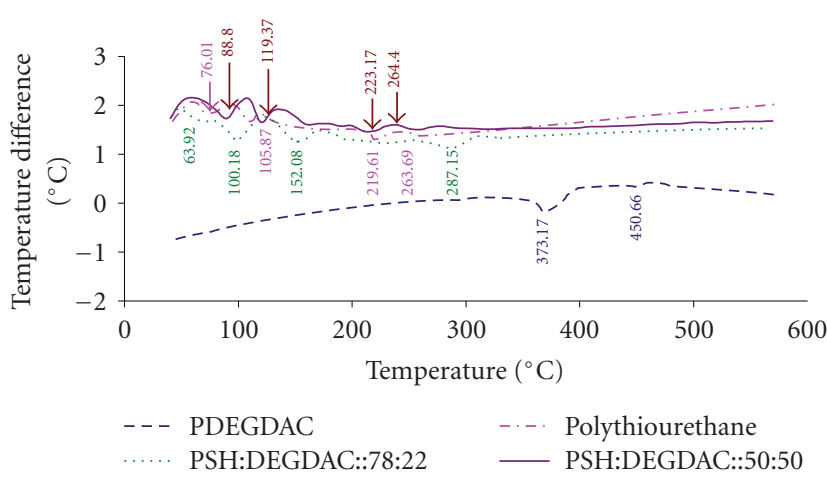

Figure 7: DTA thermogram of Polythiourethane, PDEGDAC, and combination of PSH and DEGDAC. The results indicate the formation of a new product.

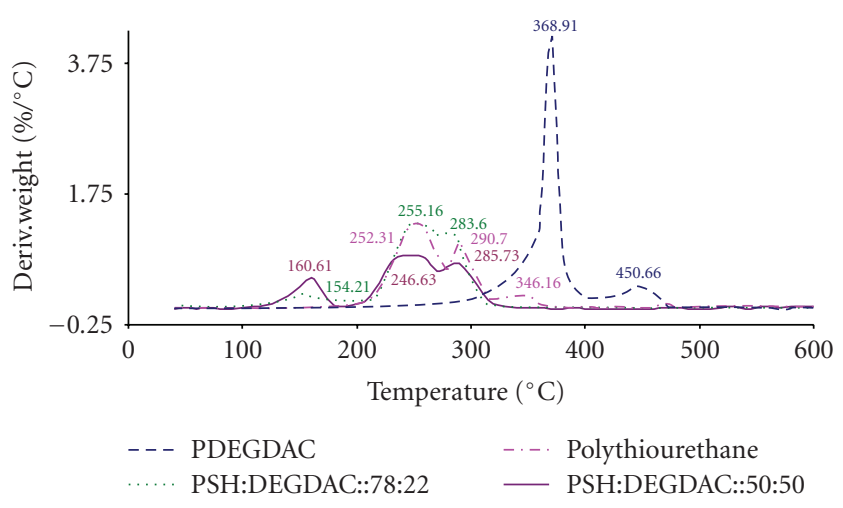

FIGURE 8: DTG thermogram of Polythiourethane, PDEGDAC, and combination of PSH and DEGDAC.

The results of DSC show that the polymer obtained from the mixtures of DEGDAC and PSH behave differently from the two components. The DSC analysis of the market sample of PDEGDAC show two endothermic peaks at $51.0^{\circ} \mathrm{C}$ and $99.2^{\circ} \mathrm{C}$ and an exothermic peak at $217.4^{\circ} \mathrm{C}$. DSC analysis indicates a $\mathrm{Tg}$ of $50^{\circ} \mathrm{C}$ to $51^{\circ} \mathrm{C}$. This agrees closely with reported [7] values. The endotherm at $99.2^{\circ} \mathrm{C}$ may be attributed to the loss of volatile components in the polymer. The exotherm at $217.4^{\circ} \mathrm{C}$ may be either ascribed to the decomposition of the polymer or due to the presence of the unreacted monomer, which undergoes polymerization with evolution of heat. The DSC analysis of polythiourethane produced from PSH and TDI indicates one endothermic peak at $99.68^{\circ} \mathrm{C}$, which can be attributed to the $\mathrm{Tg}$ of the polymer. The DSC of polymers of the mixtures of PSH and DEGDAC have endothermic peaks at $\sim 53^{\circ} \mathrm{C}, \sim 99^{\circ} \mathrm{C}$, and $\sim 184^{\circ} \mathrm{C}$. While the peaks at $\sim 53^{\circ} \mathrm{C}$ and $\sim 99^{\circ} \mathrm{C}$ are due to the glass transition of polycarbonate and polythiourethane, respectively, the peaks at $\sim 184^{\circ} \mathrm{C}$ show that there is a formation of a new structure formed by the interactions between PSH and DEGDAC from the compositions.

The results of TGA (Figure 6) of the individual polymers and the mixtures of the two monomers based on PSH and DEGDAC exhibit the following changes. 


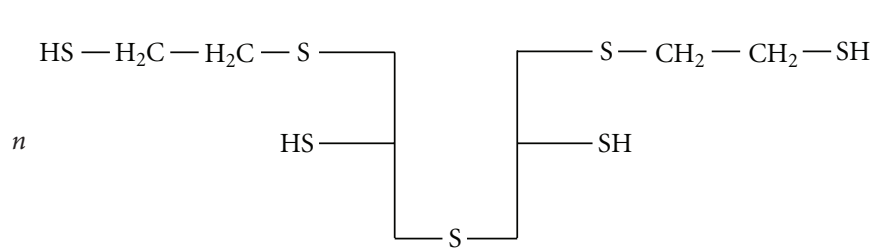

Polythiol

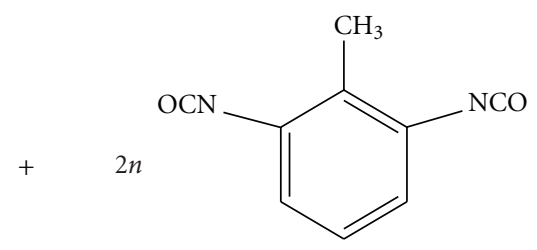

Toluene diisocyanate

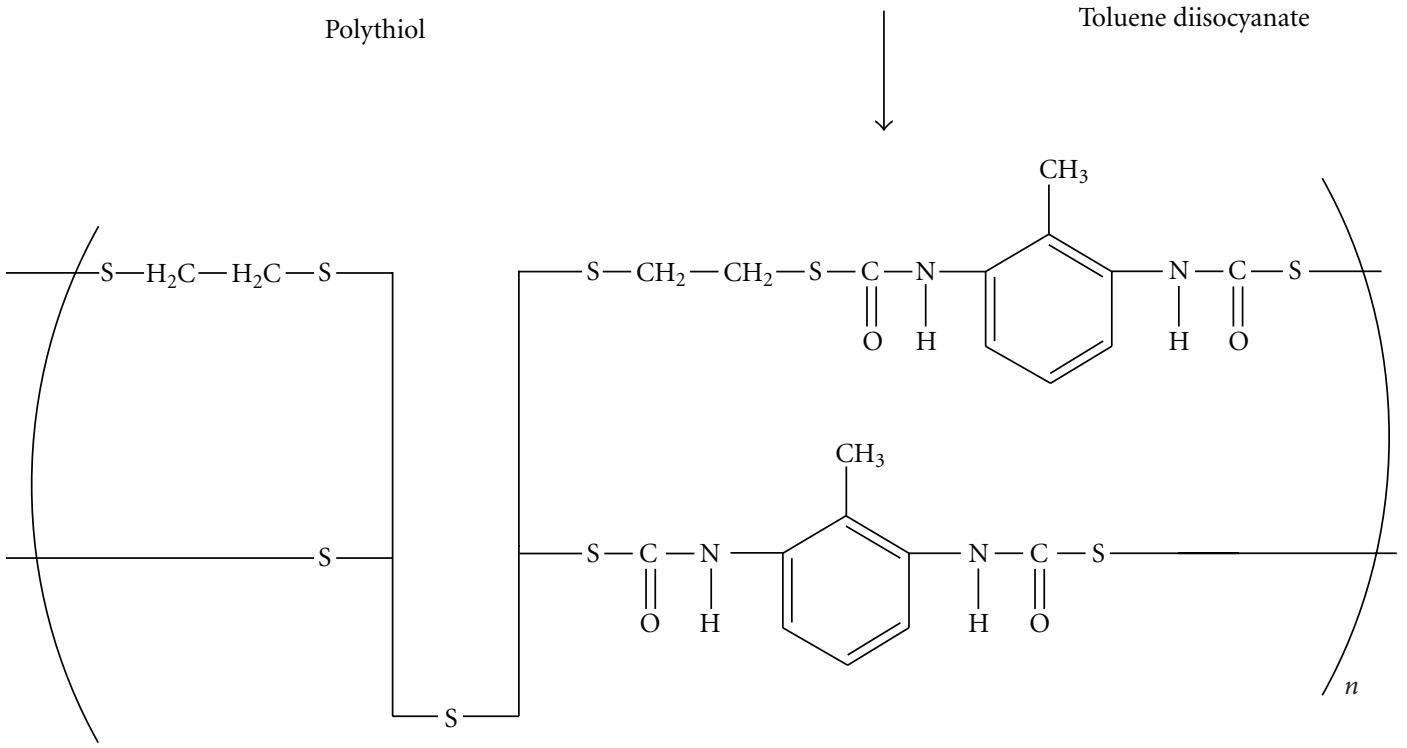

Polythiourethane

FIGURE 9: Scheme of reaction taking place between polythiol and toluene diisocyanate to form polythiourethane.

(a) PDEGDAC (curve 4) undergoes complete degradation with complete loss in weight when heated beyond $400^{\circ} \mathrm{C}$. The decomposition takes place in two stages; the major loss in weight before $400^{\circ} \mathrm{C}$ and a relatively small loss in weight beyond $400^{\circ} \mathrm{C}$.

(b) In the case of polythiourethane (curve 3), the polymer does not loose complete weight when heated upto $600^{\circ} \mathrm{C}$. The total loss in weight is $\sim 90 \%$. When compared with PDEGDAC, the polythiourethane shows degradation of the polymer at much lower temperature. Based on the amount of loss in weight, polythiourethane is more stable than PDEGDAC but looking at the temperature at which the degradation occurs, PDEGDAC would be called more stable than polythiourethane.

(c) The combinations (curves 1 and 2) of PDEGDAC and PSH falls somewhere in between the two polymers. Here, it may be noted that PSH:PDEGDAC:::78 : 22 ratio degrades completely and it is less stable than both PDEGDAC and polythiourethane. On the other hand, in the case of the ratio PSH:PDEGDAC::50 : 50 , the polymer is more stable than both of its components when seen in terms of weight loss.

From the above observations, it is evident that $50: 50$ is the most stable combination of the two components.
The results of DTA of polycarbonate with an endothermic peak at $373.17^{\circ} \mathrm{C}$ may be attributed to the decomposition of polycarbonate. The DTA results of polythiourethane show peaks at $76.01^{\circ} \mathrm{C}, 219.61^{\circ} \mathrm{C}, 105.87^{\circ} \mathrm{C}$, and $263.69^{\circ} \mathrm{C}$. The DTA results of polymers of the mixtures of PSH and DEGDAC are different from that of the polycarbonate but certain endothermic peaks have the similar pattern as that of the polythiourethane. Several peaks in the DTA and DTG show that the thermal transitions taking place at different temperatures maybe attributed to the transitions of the complex structure formed as a result of polymerisation.

From the results of analysis, it is evident that the two monomers polymerized together lead to the combination of different polymeric materials.

While the results of DTG explain the thermal stability better, the results of DTA explain the complexity of the structure of the polymer combinations. What is notable from the results is the fact that PDEGDAC as such shows two sharp peaks in DTG and DTA. The peak at $373.2^{\circ} \mathrm{C}$ in DTA is accompanied by the peak at $368.9^{\circ} \mathrm{C}$ in DTG showing that the endotherm corresponds to loss in weight due to degradation.

In the case of polythiourethane, there are four peaks in DTA and three peaks in DTG. The endotherms in DTA are not matching with the peaks in DTG indicating that before the degradation, the polymer undergoes structural transformations on heating. 


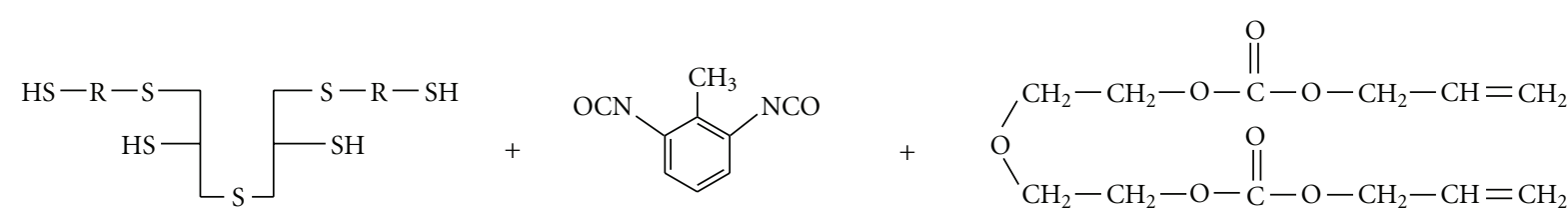

(i) Polythiol (PSH) (ii) Toluene diisocyanate (TDI)

$$
\downarrow
$$

(iii) Diethylene glycol di(allyl carbonate) (DEGDAC)

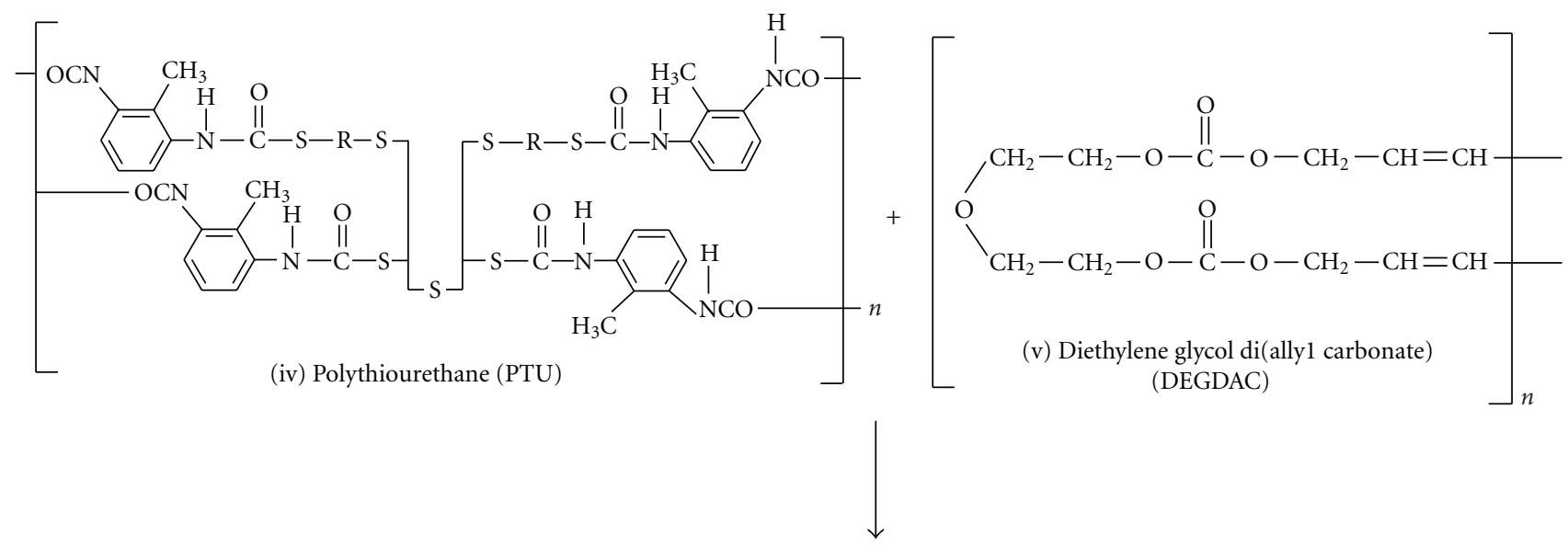

Transparent and uniform blend of PTU and DEGDAC

Figure 10: Polymerization of the composition consisting of (i) Polythiol (PSH), (ii) Toluene diisocyanate (TDI), and (iii) Diethylene glycol di(allyl carbonate) (DEGDAC). The reaction between PSH and TDI, in the presence of DBTDL results in the formation of a polythiourethane.

The combination of the two components exhibit the DTG and DTA behavior much similar to that of polythiourethane; three peaks in DTG and four peaks in DTA. Here again, the PSH:PDEGDAC::50 : 50 mixture shows better behavior than PSH:PDEGDAC::78 : 22 ratio.

Mechanism of Polymerization. In one of the earlier studies [6] the authors had already established that the polymerisation of polycarbonate can be done by gamma radiation without using any initiator [6]. This study was carried out at a semi-pilot scale demonstrating that the polymer achieved by gamma irradiation without any initiator exhibits superior properties to the material obtained by thermal polymerisation. The only mechanism that the authors could propose for this finding is the free radical initiation of DEGDAC [6].

Thermal polymerization of DEGDAC involves Diisopropyl peroxy percarbonate as a catalyst. In the present study, this catalyst was not used, as it was not found to be compatible with the compositions.

The polymerization of polythiourethane takes place by the mechanism as shown in Figure 9.

The mechanism for the polymerization of the mixtures of polythiol and DEGDAC are shown as follows and in Figure 10

$$
\begin{gathered}
D E G D A C \longrightarrow P D E G D A C \\
P S H+D E G D A C \longrightarrow P T U: P D E G D A C
\end{gathered}
$$

\section{Conclusion}

The studies involving the addition of a high refractive index additive to diethylene glycol di(allyl carbonate) have resulted in the following achievements.

(1) The use of high refractive index additive has been found to be effective in improving the properties of diethylene glycol di(allyl carbonate) and converting it into a high refractive index material suitable for optical applications.

(2) By varying the proportion of high refractive index additive, the refractive index of polycarbonate can be tailor made between 1.49-1.66 and the Abbe number can be tailor made between 26-37.

(3) Transparent, homogeneous, and clear blends with good optical properties and better thermal stability than PDEGDAC have been obtained.

(4) Use of a high refractive index additive to modify the optical properties of polymer of DEGDAC is a costeffective and easy methodology.

\section{Acknowledgments}

The authors wish to express their sincere thanks to the Management of Shriram Institute for Industrial Research, India for guidance and support on this interesting topic of research. 


\section{References}

[1] K. Othmer, Enclyclopedia of Chemical Technology, vol. 19, Wiley InterScience, New York, NY, USA, 5th edition, 2006.

[2] I. E. Muskat and F. Strain, US patent no. 2,370,567, 1945.

[3] K. Othmer, Enclyclopedia of Chemical Technology, vol. 2, Wiley InterScience, New York, NY, USA, 5th edition, 2004.

[4] I. E. Muskat and F. Strain, US patent no. 2, 403, 113, 1945.

[5] D. Freitag, G. Fenglei, and L. Morbitzer, "Routes to new aromatic polycarbonates with special material properties," Angewandte Chemie International Edition in English, vol. 30, no. 12, pp. 1598-1610, 1991.

[6] G. Suri, P. Chhabra, M. Tyagi, et al., "Properties of optical plastics prepared by gamma radiation," Journal of Polymer Materials, vol. 26, no. 1, p. 33, 2009.

[7] H. Schnell, "Chemistry and physics of polycarbonates," in Polymer Reviews, vol. 9, Interscience Publishers, New York, NY, USA, 1964.

[8] M. Verma, G. S. Jha, G. Seshadri, and R. K. Khandal, "Optical plastics-a wonder material," Journal of the Indian Chemical Society, vol. 82, no. 12, pp. 1113-1118, 2005.

[9] G. S. Jha, G. Seshadri, A. Mohan, and R. K. Khandal, "Development of high refractive index polythiourethane," $e$ Polymers, vol. 120, 2007.

[10] G. S. Jha, G. Seshadri, A. Mohan, and R. K. Khandal, "Sulphur containing plastics and its ophthalmic applications," e-Polymers, vol. 35, 2008.

[11] M. Tyagi, M. Verma, G. Seshadri, A. Malik, S. Aggarwal, and R. K. Khandal, "Metal containing polymers for optical applications," International Journal of Polymer Materials, vol. 23, p. 21, 2006.

[12] M. Tyagi, G. S. Jha, G. Seshadri, A. Malik, S. Aggarwal, and R. K. Khandal, "Metal containing polymers for optical applications part-II," International Journal of Polymer Materials, vol. 24, p. 2, 2007.

[13] M. Tyagi, G. Suri, P. Chhabra, et al., "Novel way of making high refractive index plastics; metal containing polymers for optical applications," e-Polymers, no. 100, 2009.

[14] R. K. Khandal, G. Seshadri, and G. S. Jha, "Polythiol," 2341/DEL, 2004.

[15] R. K. Khandal, G. Seshadri, and G. S. Jha, "A process for preparation of polythiol," 2342/DEL, 2004.

[16] R. K. Khandal, G. Seshadri, and G. S. Jha, "A method for the synthesis of polythiol," 100/DEL, 2005.

[17] R. K. Khandal, G. Seshadri, and G. S. Jha, "An improved lens of diethylene glycol bis allyl carbonate," PCT/IN2006/000159, WO 2007/088556 A1.

[18] American Standard Test Method for Index of Refraction of Transparent Organic Plastics ASTM D-542, 1990.

[19] American Standard Test Method D-792-00; Standard test method for density and specific gravity (Relative density) of plastics by displacement, 1995.

[20] Code of Federal Regulations Food and Drugs- Use of impact resistant lenses in eyeglasses and sunglasses, FDA-21 CFR 801.410. 

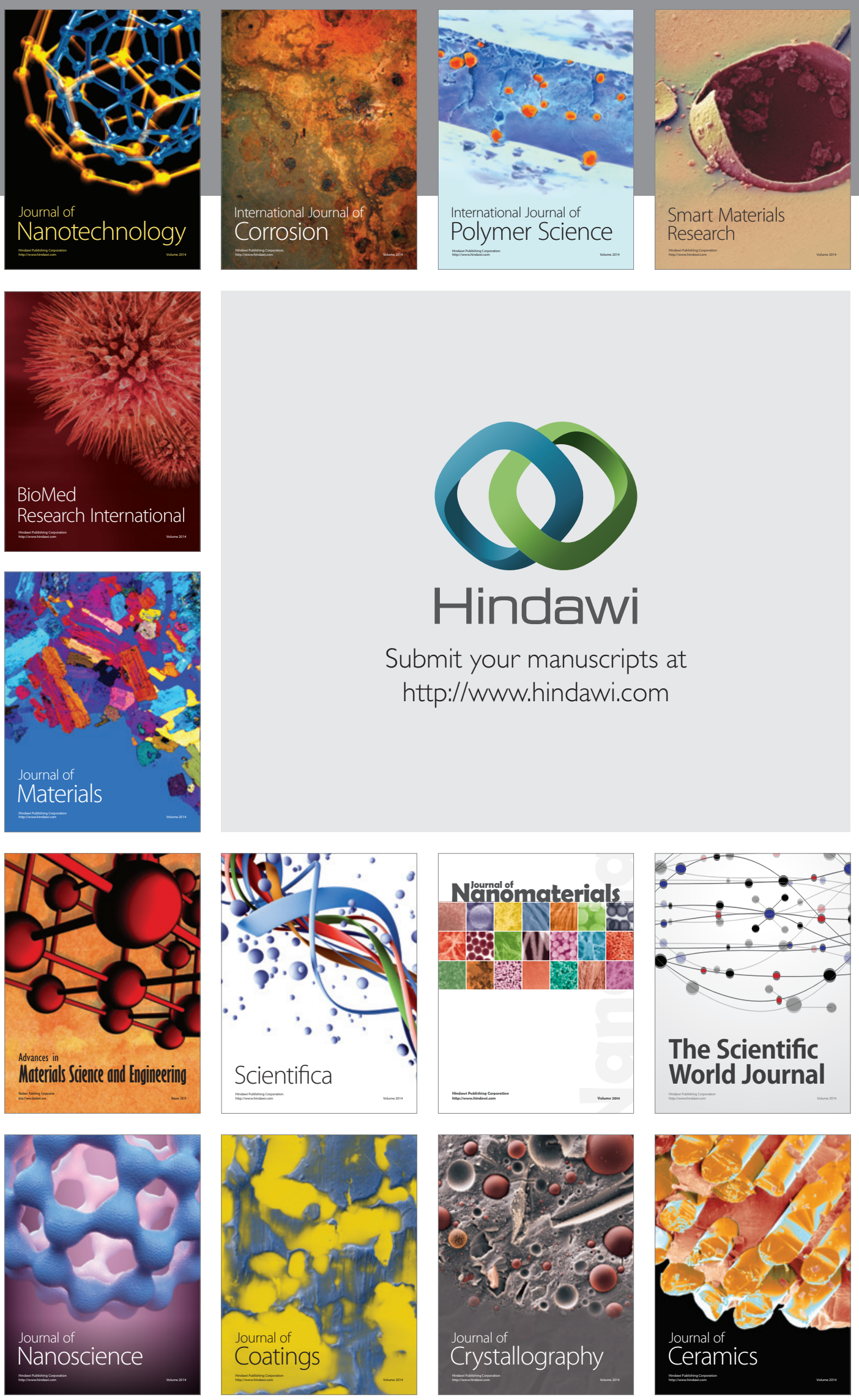

The Scientific World Journal

Submit your manuscripts at

http://www.hindawi.com

\section{World Journal}

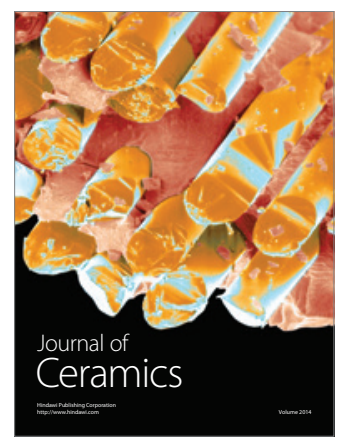

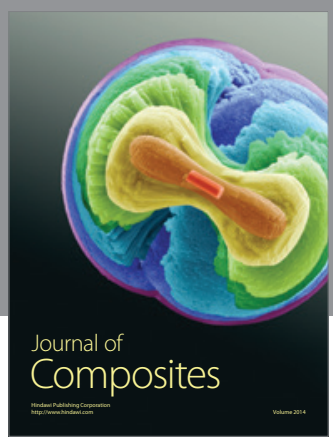
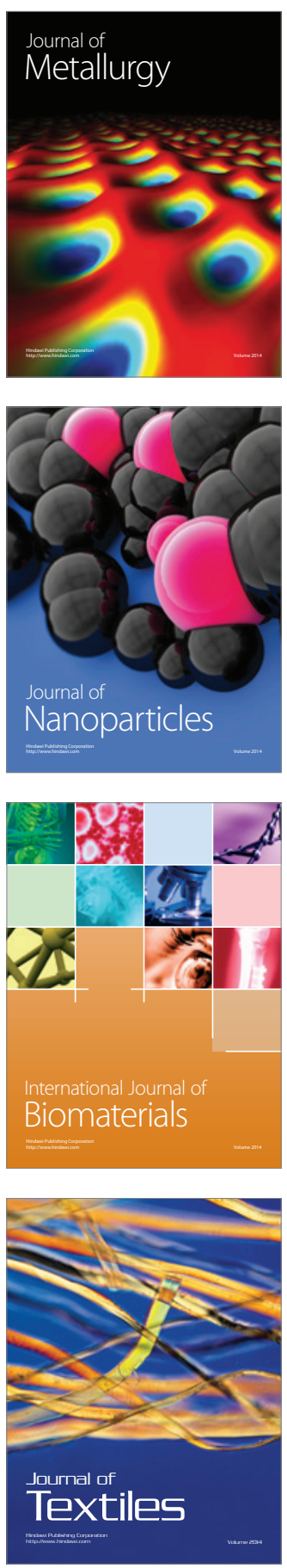